\title{
UNA NUEVA ESPECIE DE CALATOLA (ICACINACEAE) DE MÉXICO Y CENTROAMÉRICA ${ }^{1}$
}

\author{
Patricia Vera-Caletti ${ }^{2}$ \\ Colegio de Postgraduados \\ 56230 Montecillos, Edo. de México \\ México \\ Y \\ TOM WENDT \\ Plant Resources Center \\ University of Texas \\ Austin, Texas 78712, EUA
}

\section{RESUMEN}

La nueva especie Calatola uxpanapensis es un árbol de la selva alta perennifolia y mediana subperennifolia de México (Veracruz), Guatemala (El Petén e Izabal) y Belice. Esta planta ha sido confundida con $C$. laevigata Standley, especie más escasa de la vertiente del Pacífico de México, de la que difiere notablemente por su fruto anchamente elipsoide, más grande, con endocarpo profundamente reticulado y acostillado. Los dos taxa alopátricos discrepan también en detalles de las inflorescencias femeninas y masculinas, así como en sus habitats. Se incluyen datos sobre las características de campo, fenología, y distribución de la especie nueva, y también sobre la distribución de $C$. laevigata. Se especifica la presencia en México de material referible a $C$. costaricensis, y se presenta una clave para las cuatro especies del género conocidas de México.

Palabras clave: Calatola, Icacinaceae, México, Centroamérica.

\section{ABSTRACT}

The new species Calatola uxpanapensis is a tree of the evergreen and semievergreen rain forests of Mexico (Veracruz), Guatemala (Petén and Izabal), and Belize. The new species has been confused with $C$. laevigata, a rarer species of the Mexican Pacific slope, but differs markedly from that species in its larger broadly ellipsoid fruits with deeply reticulate and ribbed endocarps. The two allopatric species also differ in details of the female and male inflorescences and habitat. Data on the

1 Este trabajo forma parte de la tesis con la que la primera autora obtuvo el grado de Maestro en Ciencias, en Especialidad de Botánica, IRENAT, Colegio de Postgraduados, Montecillos.

2 Dirección actual: Área de Biología, Universidad Autónoma Chapingo, 56230 Chapingo, Edo. de México, México. 
field characteristics, phenology, and distribution of C. uxpanapensis are included, as well as distributional data for $C$. laevigata. The occurrence of material referable to $C$. costaricensis in Mexico is reported, and a key to the four known Mexican species of the genus is presented.

Key words: Calatola, Icacinaceae, Mexico, Central America.

Calatola es el tercer género más grande de la familia Icacinaceae para América, con siete especies descritas hasta ahora (Howard, 1942, 1976; Cuatrecasas, 1949). En México, las poblaciones de sus representantes se encuentran en áreas restringidas de selva húmeda y de bosque mesófilo. Por ser árboles escasos y frecuentemente altos, dioicos, con flores pequeñas, están mal representados en los herbarios; su clasificación y variación han sido poco estudiadas. Para México por lo común se han registrado dos especies: C. mollis Standley y C. laevigata Standley (Standley, 1923, 1926; Howard, 1942; Gutiérrez B., 1994). Sin embargo, la revisión del género hecha por la primera autora (Vera-Caletti, 1999) comprueba la existencia de por lo menos cuatro especies en México, añadiendo $C$. costaricensis Standley y la que aquí se reconoce como nueva a las dos arriba señaladas.

Calatola laevigata ha sido considerada como planta distribuida principalmente en las selvas altas de las tierras bajas del vertiente del Atlántico (Golfo y Caribe), desde el sur de Veracruz hasta Guatemala y Belice (Howard, 1942; Standley y Steyermark, 1949; Gutiérrez B., 1994), aunque el tipo fue colectado en el Cerro Espino cerca del litoral del Pacífico en Oaxaca. El trabajo de campo intensivo realizado por los autores, unido a la revisión de material de los principales herbarios nacionales y de los Estados Unidos, revelan que las poblaciones de la vertiente del Atlántico representan una especie distinta y nueva para la ciencia, la cual se describe a continuación.

Calatola uxpanapensis Vera-Caletti \& T. Wendt sp. nov. (Fig. 1).

A Calatola laevigata Standley fructu late ellipsoidali (4) 5-7 (7.8) x (3.2) $4-5$ (6) cm (nec ellipsoidali (2) 2.5-3.5 x 1.5-1.7 cm), putamine (3) 4.2-6.5 (7) x (3) 3.5-4.5 (4.9) cm (nec 1.5-2.5 (3.1) x 1.2-2 cm), crista aequatoria et costis putaminis crassis manifeste elevatis (nec tenuibus fere planis), inflorescentia pistillata 3-6 floribus dispersis (nec 4-10 floribus congestis), pedunculis inflorescentiarum staminatarum 4-15 mm (nec 2-3 mm) longis, pubescentia inflorescentiarum rufa (nec nivea) constanti differt.

Árboles dioicos de hasta 25 (30) $\mathrm{m}$ de altura, de hasta 50 (70) cm d.a.p., frecuentemente con contrafuertes. Corteza escamosa, de color café claro, de hasta $0.7 \mathrm{~cm}$ de grosor; brecha de la corteza laminada, con líneas amarillo-anaranjadas y de color crema, con ligero olor a jícama, albura de color amarillo muy claro, expuesta al aire toda la brecha toma una tonalidad verdosa y con más tiempo cambia a azul-violeta. Ramas muy jóvenes puberulentas con pelos aplicados, pronto glabrescentes, finamente corrugado-estriadas, con lenticelas esparcidas. Hojas dispuestas en espiral; láminas ligeramente coriáceas, estrechamente elípticas, oblongo-elípticas u obovado-elípticas, de (10.5) 14-20 (30) cm de largo y (3) 4-7 (9.5) cm de ancho, 2.7-4.1 veces más largas que anchas, margen entero y ligeramente revoluto; ápice acuminado, acumen generalmente de 1-2 cm de largo, base anchamente aguda a cuneada; venación eucamptódroma o distalmente broquidódroma, con 


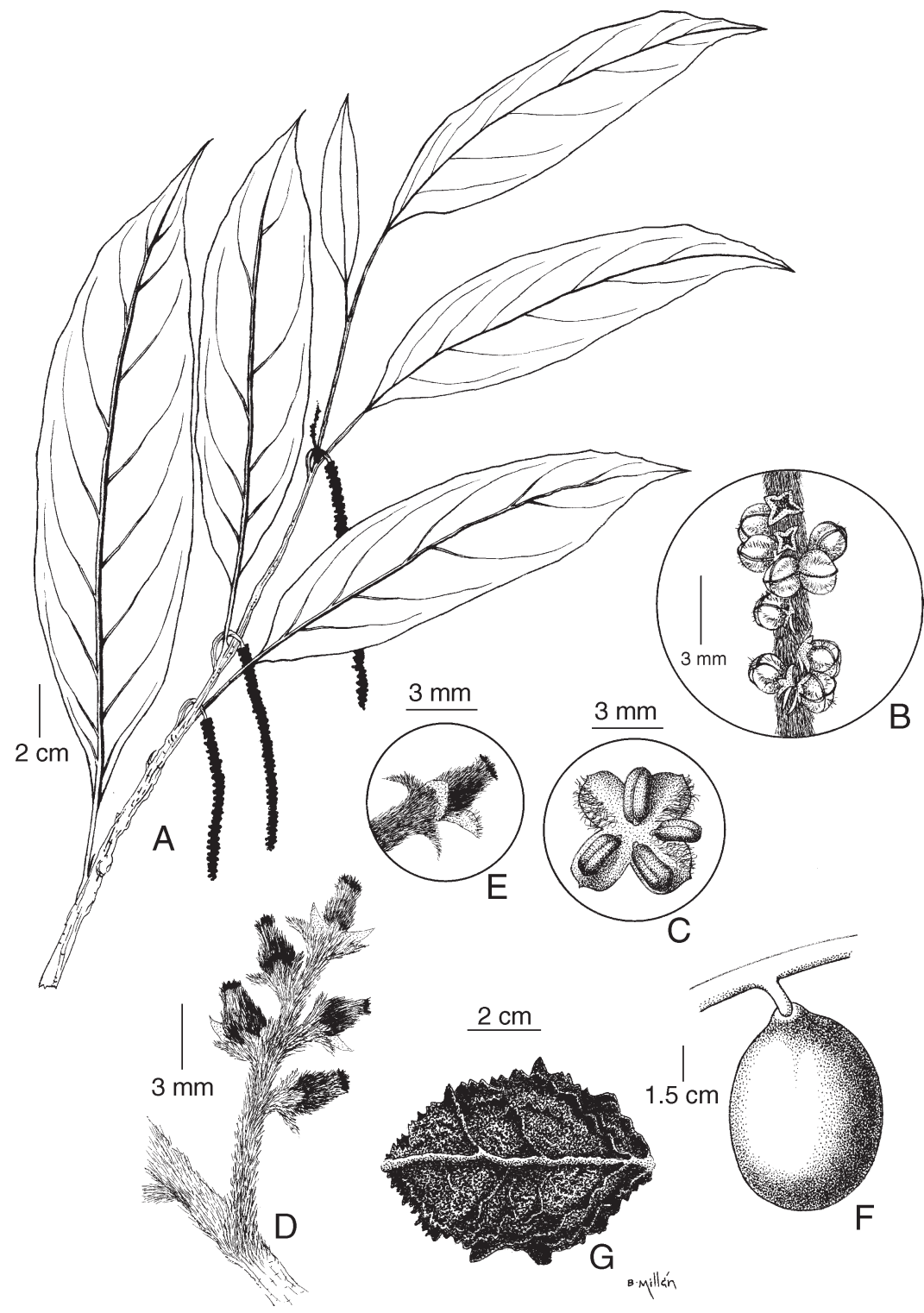

Fig. 1: Calatola uxpanapensis. A. rama con espigas masculinas; B. detalle de la espiga masculina, mostrando cáliz y pétalos valvados; $C$. flor masculina mostrando estambres; $D$. inflorescencia femenina; E. flor femenina; F. fruto; G. endocarpo, mostrando costillas y cresta principal. Ilustrado por Raúl Bravo Millán. A-C basados en Vera-Caletti y González-Ledesma 298; D-E basados en Vera-Caletti et al. 292; $\mathrm{F}$ basado en Wendt et al. 4731; G. basado en Wendt et al. 3921. 
6-10 pares de nervios secundarios muy delgados, por el haz planos o ligeramente levantados con el nervio central surcado hacia la base, por el envés marcadamente levantados; envés glabro, opaco, con el nervio medio glabro a glabrescente; haz glabro y lustroso, nervio central a veces puberulento hacia la base; pecíolo de 1.5-2.5 (4) cm de largo, acanalado, corrugado, finamente puberulento, glabrescente, de color obscuro, a veces con lenticelas; hojas adquiriendo un color negruzco al secarse.

Espigas masculinas naciendo junto a los pecíolos, una por nudo, densas, de (3) 5-12 cm de largo (excluyendo el pedúnculo) y 4-5 mm de ancho, maduración de las flores simultánea; pedúnculos de $0.4-1.5 \mathrm{~cm}$ de largo y ca. $1 \mathrm{~mm}$ de grosor, más o menos finamente puberulentos con pelos aplicados; brácteas de 0.8-1.3 mm de largo, acuminadas, el acumen 0.3-0.4 mm de largo, raquis y exterior de las brácteas finamente puberulentos con pelos rojizos o cobrizos adpresos o ascendentes. Flores actinomórficas; sépalos 4, unidos en su base, de color café claro, ligeramente coriáceos, cimbiformes, de 0.7-1.5 mm de largo y 0.3-0.5 mm de ancho, con el ápice agudo, glabros en la superficie interna, con pubescencia similar a la de las brácteas en la externa; pétalos 4, valvados, de color verde pálido, membranosos, elípticos, de 1.8-2.0 mm de largo y 1.0-1.2 mm de ancho, ápice agudo, cara interna pilosa en el nervio medio, la externa serícea sobre y alrededor del nervio medio sin cubrir toda la superficie, el margen de color más claro, venación morada; estambres 4 , de 1.0-1.5 mm de largo, alternipétalos, filamentos de 0.1-0.3 mm de largo, translúcidos, anteras basifijas, de color café obscuro, de 1.0-1.2 $\mathrm{mm}$ de largo, glabras, abriendo lateral y longitudinalmente; pistilodio ausente.

Inflorescencias femeninas (dicasios compuestos reducidos) como las espigas masculinas en posición y pubescencia, de (0.9) 1.2-1.5 cm de largo, con 3-4 (6) flores esparcidas, pedúnculo de (2.5) 3-5 mm de largo y 1.2-1.5 mm de ancho; brácteas de 1.21.4 (2) $\mathrm{mm}$ de largo, acuminadas. Pedicelos articulados, de 0.8-1.2 mm de largo. Flores actinomórficas; sépalos 4, ligeramente coriáceos, cimbiformes, de 1.0-1.5 mm de largo, la cara interna glabra, la externa cortamente rojo-serícea; pétalos 4, elíptico-ovados, equidistantes entre sí o por pares, ocasionalmente ausentes, de color café claro, de 0.8$1.0 \mathrm{~mm}$ de largo y $0.5 \mathrm{~mm}$ de ancho, pubescentes sobre el nervio medio en ambas caras, estaminodios ausentes; pistilo súpero, ovario oblongo-elipsoide, de 1.7-2.5 mm de largo y 1.4-1.7 mm de ancho, cortamente rojo-seríceo; estigma sésil, apical, fina y uniformemente multilobado, mayormente de 0.2-0.4 mm de longitud, a veces algunos lóbulos más grandes, de hasta $0.5 \mathrm{~mm}$ de largo.

Fruto una drupa, anchamente elipsoide, de color verde claro y brillante, glabro, de (4) $5-7(7.8) \mathrm{cm}$ de largo y (3.2) $4-5$ (6) $\mathrm{cm}$ de ancho, liso o moderadamente granuloso o verrugoso, base y ápice obtusos, el ápice con restos del estigma, sólo uno llegando a la madurez por inflorescencia; pedicelo de $0.8-1.5 \mathrm{~cm}$ de largo y 2-3 $\mathrm{mm}$ de ancho, finamente puberulento, glabrescente, a veces con restos de cáliz. Mesocarpo de color crema, pero cambiando a azul-violeta al contacto con el aire. Endocarpo leñoso, elipsoide, a veces globoso, de (3) 4.2-6.5 (7) cm de largo y (3) 3.5-4.5 (4.9) cm de ancho, con una cresta principal longitudinal que lo rodea y divide en dos mitades, de (3) $4-5$ (7) mm de ancho y 2-3 mm de grosor, con costillas prominentes, no afiladas, algunas redondeadas, del mismo tamaño y grosor que la cresta y con reticulaciones menores, base obtusa, ápice agudo a rostrado o a veces obtuso. 
Semilla globosa o elíptica, ocupando todo el lóculo del endocarpo; testa papirácea, de color café claro, con haces vasculares fibrosos y evidentes; endospermo abundante, de color gris traslúcido, tornándose azul-violeta al contacto con el aire, con sabor y textura de coco.

Germinación epígea, el endocarpo desprendiéndose después del alargamiento de la radícula y del hipocótilo y antes de la emergencia de los cotiledones, quedando el endospermo acuoso y de color violeta obscuro. Plántulas con el hipocótilo delgado, de ca. 7-8 cm de largo, cotiledones crasos, elípticos, de $8-8.5 \mathrm{~cm}$ de largo y 3.5-4.5 $\mathrm{cm}$ de ancho, de color amarillo claro, margen entero; nomófilos oblongo-elípticos, de 8-16 cm de largo y $2.5-4.5 \mathrm{~cm}$ de ancho, glabros, el margen dentado; cuello claramente engrosado, radícula de más de 15-16 cm de largo. Número cromosómico: $n=c a$. 14 en células madres del polen.

Nombre común: "Nuez" en la región de los Tuxtlas, Veracruz.

TIPO: MÉXICO: VERACRUZ: municipio de Minatitlán, región de Uxpanapa, 13.7 km al E de La Laguna por la terracería a Uxpanapa, luego $7.2 \mathrm{~km}$ al $\mathrm{N}$ por el camino (no completo) a Belisario Domínguez (brecha 93), selva cárstica; 17ํ20'N, 94ํ22'O., $120 \mathrm{~m}$ s.n.m., 28.IV.1984 (fr), T. Wendt, M. Ishiki I., P. Vera C., D. Woodland y B. Kenke 4731 (holotipo, MEXU; isotipos, CHAPA, ENCB, MICH, MO, NY, TEX, XAL).

Paratipos: MÉXICO: VERACRUZ: Municipio de San Andrés Tuxtla (todos de la Estación de Biología Tropical "Los Tuxtlas", U.N.A.M.): sin alt., VIII.1971 (fr. inmad.), J. I. Calzada 452 (CAS, F, GH, MEXU, MO); sin. alt., 24.IV.1972 (fl. masc.), J. I. Calzada 785 (CHAPA, F, MEXU, XAL); 200 m s.n.m., 21.X.1974 (fr.), J. I. Calzada 1593 (XAL); cerro El Vigía, 400 m s.n.m., 29.VIII.1972 (fr. inmad.), R. Cedillo 281 (CHAPA, F, MEXU, MO); El Vigía, sin alt., 15.IV.1984 (fl. fem.), R. Cedillo 2715 (CHAPA, MEXU), 15.IV.1984 (fl. masc.), 2717 (CHAPA, MEXU); cerro El Vigía, 300 m s.n.m., 7.VIII.1984 (fr. inmad.), $R$. Cedillo 2812 (CHAPA, ENCB); lote 71, límite sur, 11.IV.1985 (fl. masc.), R. Cedillo 3590 (CHAPA); cerro El Vigía, 200-500 m s.n.m., 21.V.1981 (endocarpos caídos), A. Gentry 32248 (MO); El Vigía, lote 67, 250 m s.n.m., 22.VII.1983 (fr. inmad.), G. Ibarra M. 776 (MEXU, MO); cerro El Vigía, 430 m s.n.m., 23.Il.1984 (fl. fem.), G. Ibarra M. et al. 1328 (MEXU); 200 m s.n.m., 24.II.1984 (plántulas en invernadero), G. Ibarra M. 1359 (MEXU, XAL); 200 m s.n.m., 3.IV.1984 (plántulas en invernadero), G. Ibarra M. 1458 (MEXU); 160 m s.n.m., 3.VII.1984 (plántulas), G. Ibarra M. 1846 (MEXU); camino a Lázaro Cárdenas, 3 km al NO de la estación, 250 m s.n.m., 17.V.1987 (fl. masc.), G. Ibarra M. et al. 3108 (CAS, MEXU); cerro El Vigía, 300 m s.n.m., $17 . I V .1989$ (fl. masc.), P. Vera-Caletti et al. 289 (CHAPA, TEX), 291 (CHAPA, TEX); misma localidad, 17.IV.1989 (fl. fem.), P. Vera-Caletti et al. 290 (CHAPA, TEX), 292 (CHAPA, TEX); misma localidad 17.IV.1989 (endocarpos caídos), P. Vera-Caletti et al. 293 (CHAPA, TEX), 294 (CHAPA, TEX); a $450 \mathrm{~m}$ al O de la estación, sin alt., 28.VIII.1972 (fr.), A. Villegas 42 (ENCB, F, MEXU, XAL). Municipio de Santiago Tuxtla: cerro El Vigía, 900 m s.n.m., 18.II.1967 (fr.), R. Cedillo 11 (MEXU). Municipio de Jesús Carranza: ejido "Francisco Javier Mina", a 2 km al N del Poblado Dos, 17016'N, 94040'O., 120 m s.n.m., 4.V.1982 (endocarpos caídos), M. Vázquez T. et al. 2452 (CHAPA, TEX); misma localidad, 24.X.1982 (fr.), M. Vázquez T. et al. 2562 (CHAPA, XAL); misma localidad, 31.Ill.1987 (fl. masc.), P. Vera-Caletti et al. 275 (CHAPA, TEX); misma localidad, 19. IV.1989 (fl. masc.), 
P. Vera-Caletti y M. González-Ledesma 297 (CHAPA, TEX), 298 (CHAPA, TEX); misma localidad, 29.IV.1982 (endocarpos caídos), T. Wendt et al. 3921 (CHAPA). Municipio de Minatitlán: localidad tipo, 29.III.1987 (estéril), P. Vera-Caletti et al. 274 (CHAPA, TEX); misma localidad, 20.IV.1989 (estéril), P. Vera-Caletti y M. González-Ledesma 300 (CHAPA, TEX), 300-A (CHAPA, TEX), 301 (CHAPA, TEX.); misma localidad, $20 . I V .1989$ (endocarpos caídos), P. Vera-Caletti y M. González-Ledesma 302 (CHAPA, TEX).

BELICE: DIST. TOLEDO: Temash River, alt. 50 ft., 8.XI.1933 (fr.), W. A. Schipp S446 (A, F, GH, MEXU, MICH, MO), alt. 200 ft., 17.VI.1935 (fl. masc.), W. A. Schipp 1366 (A, F, GH, MEXU, MICH, MO).

GUATEMALA: DEPTO. IZABAL: $6 \mathrm{~km}$ al NO de Cadenas [= Puerto Méndez = Modesto Méndez], 10.VIII.1967 (fr), E. Contreras 7004 (DS, F, LL). DEPTO. EL PETÉN: km 9.1 brecha Petexbatun-Chinaja, 20.V.1965 (fl. masc.), E. Contreras 5404 (LL); La Cumbre, al E del km. 141, 30.V.1975 (fl. masc.), C. L. Lundell y E. Contreras 19370 (LL).

Calatola uxpanapensis se conoce del estado de Veracruz, México, en las regiones de Los Tuxtlas y Uxpanapa, así como de Guatemala (El Petén e Izabal) y Belice (Fig. 2). En México se encuentra en selva alta perennifolia o en selva mediana subperennifolia, acompañada de representantes de géneros como Brosimum, Dialium, Ficus, Lonchocarpus y Sloanea entre otros, en altitudes de 100-900 m., en suelos profundos o en zonas kársticas. En Centroamérica, los datos de colecta indican selvas altas, "zapotales" (selvas de Manilkara zapota (L.) P. Royen), y "corozales" (palmares de Orbignya cohune (C. Mart.) Standley). No se puede descartar la posibilidad de que la aparente ausencia de la especie nueva en la región entre el sur de Veracruz y el sur del Petén pueda deberse a la falta de conocimiento suficiente sobre la flora de esta región. Sin embargo, Wendt (1998) presenta ejemplos de árboles con distribuciones geográficas semejantes a la aquí señalada para $C$. uxpanapensis. Dichas especies aparentemente se restringen a selvas que prosperan en comarcas de muy alta precipitación, como son las de las regiones de Los Tuxtlas, Uxpanapa, y el sur del Petén.

En Veracruz, la especie nueva florece de febrero a mayo y presenta frutos maduros de septiembre a febrero. Para Centroamérica, hay colectas con flor de mayo a junio y con frutos de agosto a noviembre. En el campo en México no se registró ningún uso. Para Belice, en las etiquetas de los ejemplares, se menciona la semilla como comestible.

La confusión registrada entre Calatola uxpanapensis y $C$. laevigata radica principalmente en la semejanza vegetativa de las dos especies, en la falta de colectas anteriores de fruto maduro de $C$. laevigata, y en la escasez de esta última. El tipo de $C$. laevigata (citado más adelante) incluye sólo ramitas con hojas e inflorescencias estaminadas. El trabajo de campo en el Cerro Espino realizado por la primera autora permitió establecer claramente las características e identidad de esta especie, la cual se distribuye solamente en la vertiente del Pacífico y partes colindantes de los declives del Golfo de la Sierra Madre de Chiapas, en forma aparentemente muy discontinua desde Chiapas hasta Jalisco (Fig. 2), siendo una planta bastante escasa en toda esta región. Se encuentra en selva mediana subperennifolia y subcaducifolia y bosque mesófilo de 900 a 1900 m s.n.m. Por lo tanto, parece ser que las dos especies son completamente alopátricas (Fig. 2) y discrepan en sus intervalos altitudinales y en sus habitats. 
Las diferencias morfológicas más notables entre ambas se encuentran en el tamaño del fruto, así como en el tamaño y forma del endocarpo (Figs. 1, 3). El fruto maduro de Calatola uxpanapensis es anchamente elipsoide, normalmente de 5-7 cm de largo por 4$5 \mathrm{~cm}$ de ancho, mientras el de $C$. laevigata es mucho más pequeño y delgado, de $3-3.5 \mathrm{~cm}$ de largo por $1.5-1.7 \mathrm{~cm}$ de ancho. El endocarpo de $C$. uxpanapensis es de (3)4-5(7) cm de largo y (3.0)3.5-4.5(4.9) cm de ancho, leñoso, y con la cresta principal longitudinal, las costillas, y las reticulaciones fuertemente levantadas y prominentes; en C. laevigata el endocarpo es de (1.5)2-2.5(3.1) cm de largo y (1.2)1.5(2.0) cm de ancho, de consistencia semejante a corcho, la cresta principal y las costillas, de estar presentes, se observan tenues y apenas levantadas. Vale la pena mencionar que a veces se encuentran, abajo de un árbol de C. uxpanapensis, algunos endocarpos caídos inmaduros que asemejan en cierto grado a los de $C$. laevigata, hecho que puede complicar la identificación de las especies, especialmente en el herbario. Sin embargo, las observaciones de campo de los autores indican claramente que los árboles femeninos de $C$. uxpanapensis siempre producen frutos y endocarpos maduros de tamaño más grande y que $C$. laevigata los tiene constantemente más pequeños.

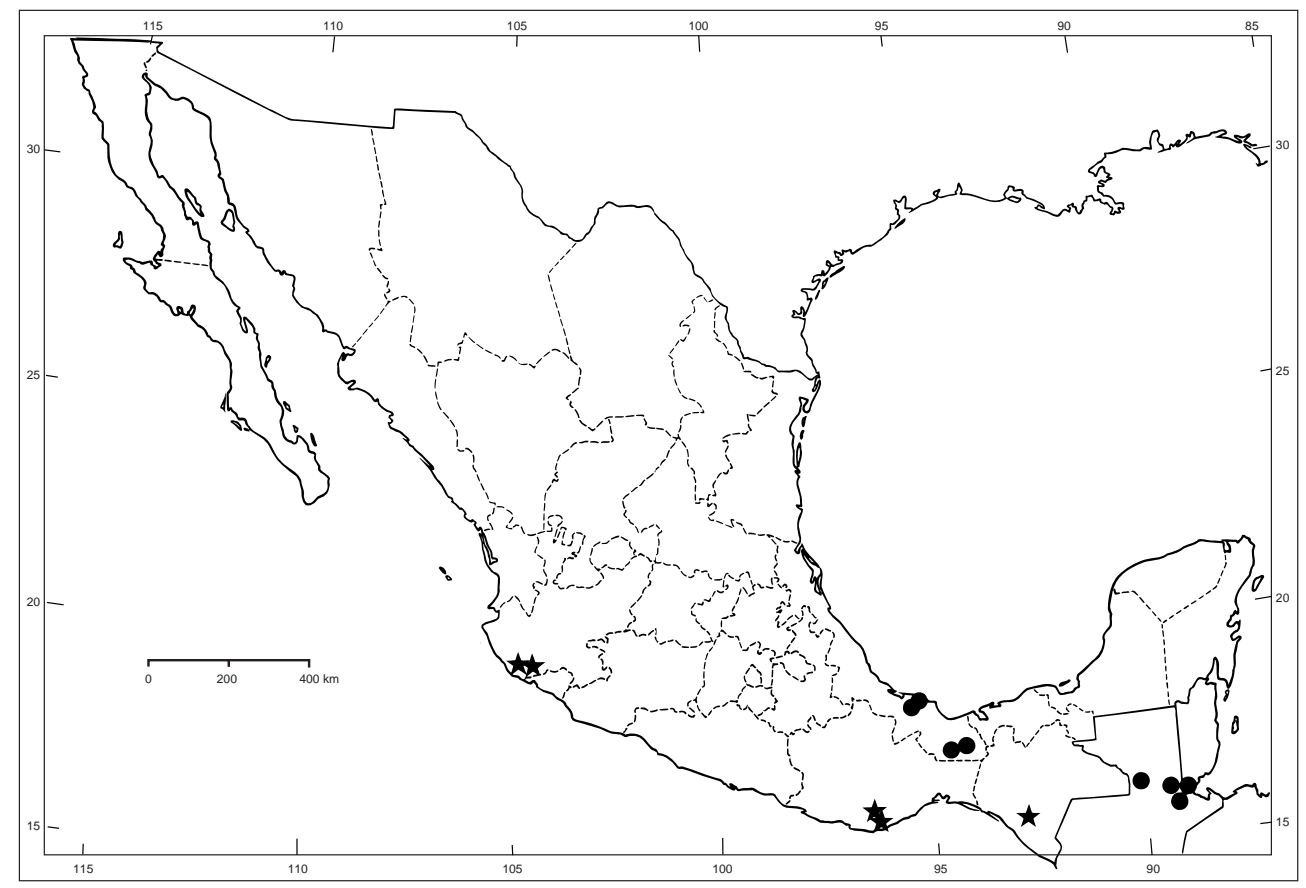

Fig. 2: Distribución conocida de Calatola uxpanapensis (círculos) y C. laevigata (estrellas) en México y Centroamérica. 


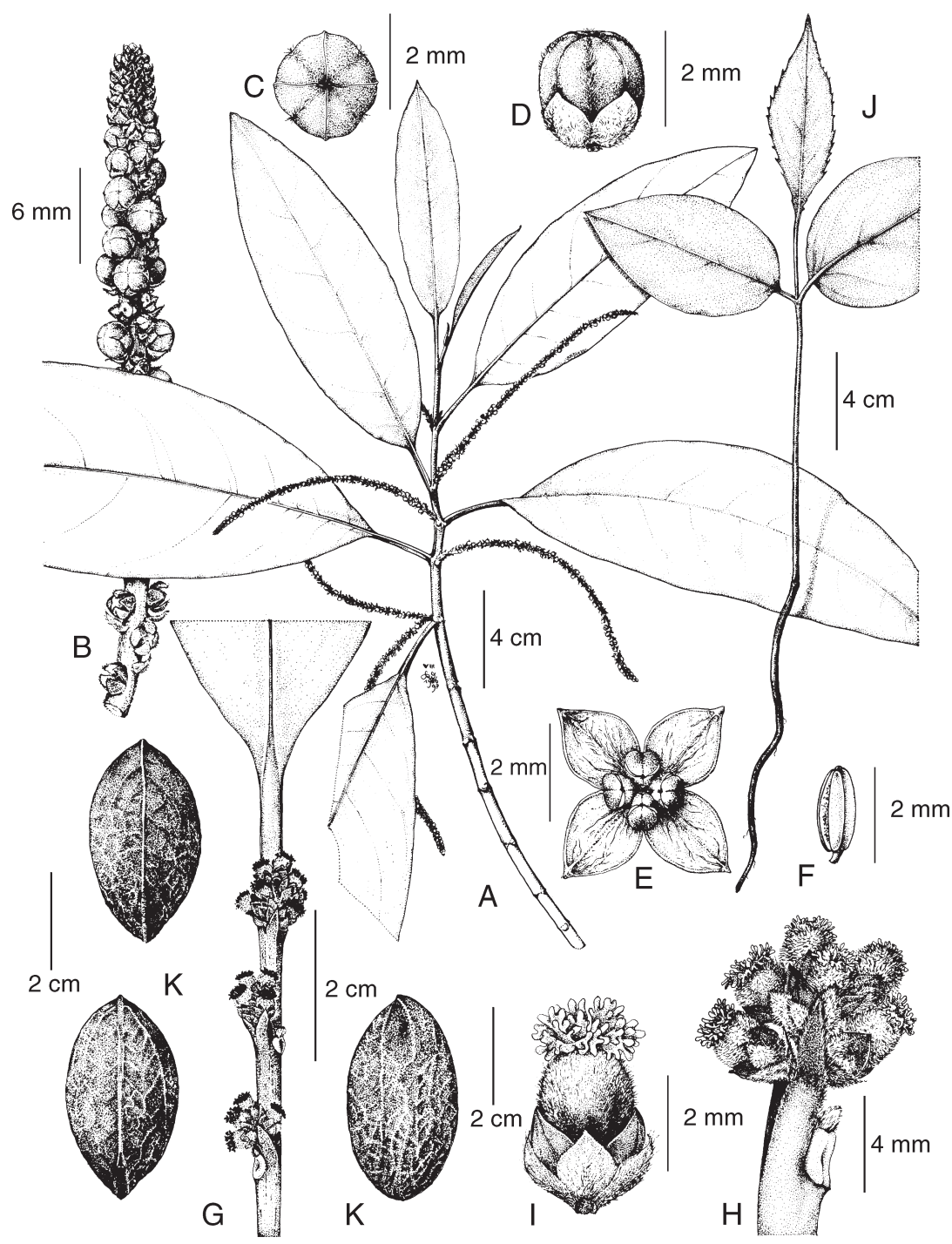

Fig. 3: Calatola laevigata. A. rama con espigas masculinas; B. parte de la espiga masculina a la izquierda mostrando botones justo antes de abrir; C. botón de flor masculina visto de arriba, mostrando pétalos valvados; D. botón de flor masculina visto de perfil, mostrando cáliz y corola; E. flor masculina abierta vista de arriba, mostrando pétalos y estambres; F. antera; G. parte de una rama con inflorescencias femeninas; $H$. inflorescencia femenina; I. flor femenina; J. plántula; $K$. endocarpos. Ilustrado por Karin Douthit para la Flora NovoGaliciana y reproducido con permiso del Herbario de la Universidad de Michigan. A y B basados en McVaugh 23224; C, D, E, F, J y K basados en Vera-Caletti y González Ledesma 303; G, H e I basados en González-Ledesma y Catalán H. 595. 
Otras diferencias importantes se encuentran en las inflorescencias. Nuestro material indica que los dicasios femeninos de Calatola uxpanapensis son de 3-5 (6) flores esparcidas, los sépalos miden 1-1.5 mm de largo, y el pedúnculo delgado es de (2.5) 3-5 mm de largo, mientras los dicasios pistilados de C. laevigata son de 4-6 (10) flores muy apretadas, los sépalos 0.7-1 mm de largo y el pedúnculo grueso ca. $2 \mathrm{~mm}$ de largo. Además, los pedúnculos de las espigas masculinas son de 4-15 mm de largo en $C$. uxpanapensis, mientras son de apenas 2-3 $\mathrm{mm}$ de largo en $C$. laevigata. Finalmente, la pubescencia de las inflorescencias masculinas y femeninas de $C$. uxpanapensis es de un aspecto rojizo o cobrizo, mientras la de $C$. laevigata siempre es blancuzca.

Estudios cromosómicos en células madre de polen de tres colectas de Calatola uxpanapensis (Vera 275, 289, 298) dieron resultados no suficientemente claros para poder determinar si el número cromosómico de cada una es $n=13 \circ n=14$. Sin embargo, recuentos definitivos de $n=14$ para C. laevigata y C. mollis (Vera-Caletti, 1999) sugirieron que la especie nueva probablemente tiene el mismo número.

A continuación se citan los ejemplares estudiados de Calatola laevigata:

MEXICO: JALISCO: Municipio de Autlán: Estación Científica Las Joyas, $1 \mathrm{~km}$ al S de Corralitos, cañada del Alentisco, 1900 m s.n.m., 24.VII.1992 (fr), R. Cuevas y L. Guzmán 4377 (CHAPA, TEX). Municipio de Cuautitlán: Sierra de Manantlán, 1-4 km abajo de "La Cumbre", cerca del camino de madereros entre El Chante y Cuzalapa, arriba de El Durazno, $19^{\circ} 32^{\prime} \mathrm{N}, 104^{0} 14^{\prime} \mathrm{W}, 1500-1900$ m s.n.m., 22-23.III.1965 (fl. masc.), R. McVaugh 23224 (ENCB, MICH). Municipio de Casimiro Castillo: 1-2 km al E de El Tecolote, 1400 m, 26.Il.1987 (fr), R. Cuevas y M. Rosales 1864 (WIS). OAXACA: Municipio de San Pedro Pochutla: finca El Calvario, vertiente E del Cerro Espina, 800 m s.n.m., 10.XII.1993 (fl. fem., fr. y endocarpos caídos), M. González-Ledesma y C. Catalán H. 595 (CHAPA, MICH, TEX); Cafetal San Carlos, Cerro Espino, $30 . I X .1917$ (fl. masc.), B. P. Reko 3440 (holotipo US!); Cafetal Calvario, 700 m s.n.m., $15 . X I I .1917$ (fr. inmad.), B. P. Reko 3728 (US); Cerro Espino, 1 km al E de la Finca Montecristo, 1200 m s.n.m., 15.IX.1989 (fl. masc. y endocarpos caídos), P. VeraCaletti y M. González-Ledesma 303 (CHAPA, MICH, TEX). Municipio de Pluma Hidalgo: Pluma Hidalgo, Cerro La Pasionaria, alt. 4500 pies, 14.III.1966 (fr. y endocarpos caídos), T. MacDougall 476-S (US). CHIAPAS: Valles de Cuztepeques, Finca El Gadow, XII.1953, F. Miranda 7814 (MEXU, US), 7816 (MEXU, US).

A continuación se proporciona una clave para distinguir entre las cuatro especies de Calatola actualmente conocidas de México, incluyendo a C. uxpanapensis.

1 Hojas maduras por lo general de $14-20 \mathrm{~cm}$ de largo y $4-8 \mathrm{~cm}$ de ancho, completamente glabras o cuando más con pelos aplicados muy esparcidos sobre la vena central en el envés; espigas estaminadas de $4-12 \mathrm{~cm}$ de largo; endocarpos con la cresta principal longitudinal, las costillas y las paredes de las reticulaciones gruesas y más o menos redondeadas, no afiladas. (2)

2 Frutos maduros elipsoides, $2-3.5 \mathrm{~cm}$ de largo; endocarpos de menos de $3 \mathrm{~cm}$ de largo, de textura semejante a corcho, casi sin costillas ni cresta principal evidentes, normalmente casi lisos; pedúnculos de las espigas masculinas de 2-3 mm de largo; pubescencia de las inflorescencias de color blancuzco C. laevigata 
2 Frutos maduros anchamente elipsoides, 4-7 cm de largo; endocarpos de más de $4 \mathrm{~cm}$ de largo, leñosos, con costillas y cresta principal bien desarrolladas; pedúnculos de las espigas masculinas de 4-15 mm de largo; pubescencia de las inflorescencias de color rojizo o cobrizo C. uxpanapensis

1 Hojas maduras por lo general de $16-30 \mathrm{~cm}$ de largo y 8-12 cm de ancho, por el envés densamente pubescentes o con mechones de pelos erectos en las axilas de las venas laterales (raras veces solamente con pelos aplicados); espigas estaminadas de (9) 14$26 \mathrm{~cm}$ de largo; endocarpos con la cresta principal longitudinal, las costillas y las paredes de las reticulaciones delgadas y afiladas. (3)

3 Envés de la hoja madura densamente pubescente con pelos erectos sobre toda la venación y por lo general sobre la superficie, aterciopelado al tacto; la brecha de la corteza se oxida a un color azuloso inmediatamente al cortarse; frutos densamente puberulentos en la madurez; endocarpo con la parte más alta de la cresta principal 2-4 $\mathrm{mm}$ de alto C. mollis

3 Envés de la hoja con pelos erectos sólo sobre la vena central y/o en las axilas y partes inferiores de las venas laterales; la brecha de la corteza se oxida a un color azuloso lenta (ca. 5 minutos) e irregularmente; frutos ligeramente puberulentos o glabrescentes en la madurez; endocarpo con la parte mas alta de la cresta principal 4-8 mm de alto

C. costaricensis

Calatola mollis es una especie endémica de Puebla (holotipo de Zacatlán, F. Salazar s. n., US!) y del centro de Veracruz, donde habita bosques mesófilos a los 600-1100 m s.n.m. Las poblaciones mexicanas de $C$. costaricensis han sido incluidas en varios trabajos sobre la flora de Mexico dentro del concepto de C. mollis (e.g., Gutiérrez B., 1994; Ibarra M. y Sinaca C., 1987) o de C. laevigata (e.g., Breedlove, 1986), aunque su relación es obviamente más estrecha con C. mollis (Vera-Caletti, 1999). Ibarra M. y Sinaca C. (1995) utilizan el nombre $C$. costaricensis para las plantas de la Estación de Biología Tropical "Los Tuxtlas", a raíz de los estudios (en proceso en aquel entonces) de la primera autora del presente trabajo. Las plantas mexicanas del complejo C. mollis- $C$. costaricensis del sur de Veracruz (Los Tuxtlas), este de Oaxaca (Chimalapa), y Chiapas son claramente referibles a C. costaricensis, con base en las características señaladas en la clave y la forma de ornamentación del endocarpo; la distribución total de esta especie se extiende hasta Costa Rica (holotipo de Heredia, P. C. Standley \& J. Valerio 50000, US!) y Panamá. Sin embargo, existen poblaciones del norte de Oaxaca y del centro de Veracruz referibles a $C$. costaricensis en cuanto a las características del fruto pero con pubescencia foliar muy semejante a la de $C$. mollis, especie que también se encuentra en el centro de Veracruz. Además, en Centroamérica $C$. costaricensis es variable y posiblemente incluye más de una especie. Vera-Caletti (1999) discute estos problemas e indica que hacen falta más estudios sobre estas plantas. En México, C. costaricensis se encuentra en selvas altas y medianas perennifolias y bosque mesófilo a los 200-1300 m s.n.m. En la región de Los Tuxtlas, Veracruz, se encuentra geográfica y altitudinalmente muy cerca de $C$. uxpanapensis, pero las dos especies no son ecológicamente simpátricas. 


\section{AGRADECIMIENTOS}

Agradecemos al M.C. Manuel González Ledesma por su apoyo en el terreno y en el laboratorio; a Heriberto Hernández González por su valiosa ayuda en el trabajo de campo; a Raúl Bravo Millán por el dibujo de C. uxpanapensis; al Dr. William Anderson, Karin Douthit y al Herbario de la Universidad de Michigan por el permiso de publicar la ilustración de $C$. laevigata; al Dr. Stephen D. Koch por la revisión del escrito; a Fernando Chiang por proporcionar datos del herbario MEXU; al CONACyT por su apoyo económico; a dos revisores anónimos por sus valiosas sugerencias; y a los curadores de los herbarios citados por las facilidades otorgadas para la consulta de los ejemplares mencionados en el trabajo.

\section{LITERATURA CITADA}

Breedlove, D. E. 1986. Listados florísticos de México. IV. Flora de Chiapas. Instituto de Biología, Universidad Nacional Autónoma de México, México, D.F. v + 246 pp.

Cuatrecasas, J. "1948" (1949). Studies in South American plants, I. Lloydia 11: 185-225.

Gutiérrez B., C. 1994. Icacinaceae. Flora de Veracruz 80: 1-16.

Howard, R. A., 1942. Studies of the Icacinaceae IV. Consideration of the New World genera. Contr. Gray Herb. 142: 3-60.

Howard, R. A. 1976. Flora of Panama. Icacinaceae. Ann. Missouri Bot. Gard. 63: 399-418.

Ibarra M., G., y S. Sinaca C. 1987. Listados florísticos de México. VII. Estación de Biología Tropical Los Tuxtlas, Veracruz. Instituto de Biología, Universidad Nacional Autónoma de México, México, D.F. 51 pp.

Ibarra M., G., y S. Sinaca C. 1995. Lista florística comentada de la Estación de Biología Tropical "Los Tuxtlas", Veracruz, México. Revista Biol. Trop. 43: 75-115.

Standley, P.C. 1923. Trees and shrubs of Mexico (Oxalidaceae-Turneraceae). Contr. U. S. Nat. Herb. 23: $517-848$.

Standley, P. C. 1926. The genus Calatola. Journ. Wash. Acad. Sci. 16: 413-418.

Standley, P. C., y J. A. Steyermark. 1949. Flora of Guatemala, Part 6. Fieldiana, Bot. 24(6): 1-438.

Vera-Caletti, P. 1999. Revisión del género Calatola (Icacinaceae) en México. Tesis de Maestría, Colegio de Postgraduados, Montecillos, México. $107 \mathrm{pp}$.

Wendt, T. 1998. Composición, afinidades florísticas y orígenes de la flora arbórea del dosel de los bosques tropicales húmedos de la vertiente mexicana del Atlántico. In: Ramamoorthy, T. P., R. Bye, A. Lot y J. Fa (eds.). Diversidad biológica de México: orígenes y distribución. Instituto de Biología, Universidad Nacional Autónoma de México. México, D.F. pp. 581-664. 